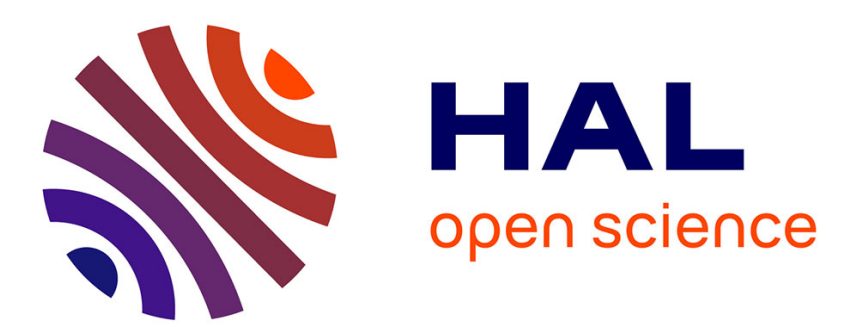

\title{
Experimental correlation of rheological relaxation and interface healing times in welding thermoplastic PEKK composites
}

Julien Avenet, Thomas A Cender, Steven Le Corre, Jean-Luc Bailleul, Arthur Lévy

\section{To cite this version:}

Julien Avenet, Thomas A Cender, Steven Le Corre, Jean-Luc Bailleul, Arthur Lévy. Experimental correlation of rheological relaxation and interface healing times in welding thermoplastic PEKK composites. Composites Part A: Applied Science and Manufacturing, 2021, 149, pp.106489. 10.1016/j.compositesa.2021.106489 . hal-03266220

\section{HAL Id: hal-03266220 https://hal.science/hal-03266220}

Submitted on 21 Jun 2021

HAL is a multi-disciplinary open access archive for the deposit and dissemination of scientific research documents, whether they are published or not. The documents may come from teaching and research institutions in France or abroad, or from public or private research centers.
L'archive ouverte pluridisciplinaire HAL, est destinée au dépôt et à la diffusion de documents scientifiques de niveau recherche, publiés ou non, émanant des établissements d'enseignement et de recherche français ou étrangers, des laboratoires publics ou privés. 


\title{
Experimental correlation of rheological relaxation and interface healing times in welding thermoplastic PEKK composites
}

\author{
Julien Avenet ${ }^{\mathrm{a}, \mathrm{b}}$, Thomas A. Cender ${ }^{\mathrm{c}}$, Steven Le Corre ${ }^{\mathrm{a}}$, Jean-Luc Bailleul ${ }^{\mathrm{a}}$, \\ Arthur Levy ${ }^{\mathrm{a}, *}$ \\ ${ }^{a}$ Université de Nantes, CNRS, Laboratoire de thermique et énergie de Nantes, LTeN, UMR \\ 6607, F-44000 Nantes, France. \\ ${ }^{b}$ IRT Jules Verne, Chemin du Chaffault, 44340 Bouguenais, France \\ ${ }^{c}$ Center for Composite Materials, University of Delaware, Newark, DE 19716, USA
}

\begin{abstract}
The evolution of polymer welding time to join the interface of thermoplastic Polyetherketoneketone (PEKK) matrix composites was characterized. Using a creep-recovery test protocol to analyse the evolution of rheological behavior, it was shown that for temperatures above the melting point $\left(T>360^{\circ} \mathrm{C}\right)$, the polymer visco-elastic relaxation time increases exponentially with time-indicating polymer crosslinking or branching. To confirm that relaxation time is proportional to interface healing time, the rheological results were compared to the fracture energy release rate $\left(G_{1 c}\right)$ of samples prepared on a custom bench which is capable of isothermally weld composite samples for controlled time intervals. The welding time corresponds directly to the relaxation time. Furthermore, the thermal history that the polymer experienced results in cross linking and influences the welding time. The implication is that temperature exposure from prior processing steps (IR oven pre-heating or autoclave consolidation) can limit the ability to weld components in a predictable way.
\end{abstract}

Keywords: A. Thermoplastic resin, B. Adhesion, D. Rheology, E.

\footnotetext{
* Corresponding author

Email addresses: julien.avenet@univ-nantes.fr (Julien Avenet), tcender@udel.edu (Thomas A. Cender), steven.le-corre@univ-nantes.fr (Steven Le Corre), jean-luc.bailleul@univ-nantes.fr (Jean-Luc Bailleul), arthur.levy@univ-nantes.fr (Arthur Levy )
}

Preprint submitted to Composites Part A: Applied Science and ManufacturingJune 14, 2021 
Joints/joining

\section{Introduction}

Composite aerospace structures are often composed of large monolithic components because composites are uniquely vulnerable in the vicinity of fasteners $[1,2]$. Where possible, bonding without fasteners is a more desirable joining approach. Thermoplastics matrix composites offer the ability to be joined by welding, which has several advantages in terms of materials and manufacturing processes. Due to the lack of chemical crosslinks, thermoplastic polymers can be melted and remolded. In the melt state, thermoplastics molecular chains can relax and slide out of their entanglements. During welding, the process of melted thermoplastic molecular chains diffusing across the interface (to form new entanglements) is known as healing. Aromatic thermoplastics, such as those of the PAEK family, are good candidates for many high performance engineering applications. In particular, carbon fiber reinforced PEKK could be used in many aerospace structures because of its high stiffness and strength [3], high glass transition temperature, and high chemical resistance in the crystalline state $[4,5]$.

These same properties which make PAEK polymers high performance, also contribute to it's difficultly in rapid welding processes. The process of thermoplastic welding of composite parts was outlined well by Lee \& Springer [6]. For their early generation of APC-2 PEEK polymer composites, they reported times to reach full weld to be well over an hour. Such long processes times are completely incompatible with rapid welding processes, such as resistance or induction welding, or even the automated fiber placement process. However, such processes are commonly used with PEEK and PEKK matrix composites with mixed or good result. In this article, we outline a methodology to characterize the weldability of thermoplastic PEKK composites and demonstrate that polymer degradation (resulting from thermal history) will lengthen the time required-thus limit the ability-to weld a polymer interface. 
To weld two thermoplastic composite components, the interface must be heated to a temperature near or above the 'melting-point' while the interfaces are in contact [2]. The mechanism for heating varies [7]: hot gas, infrared, friction, electrical resistance, induction-the goal is to localize the application of heat to the joint interface, as to avoid melting and deconsolidating the bulk part.

Since surfaces are never perfectly smooth, the problem of achieving intimate contact involves the deformation of the interface under pressure $[6,8]$. At elevated temperature, the polymer is in a viscous state, so by applying pressure, roughness at the interface will be filled by squeeze flow of the polymer. Without sufficient pressure and temperature, achieving full intimate contact could be a rate limiting step. Continuous welding processes such as tape placement, ultrasonic or induction welding, apply high pressure locally to ensure a full intimate contact is achieved quickly (within the process time scale) [9].

Once intimate contact is achieved, healing often appears as the rate limiting phenomenon in the case of fast or continuous welding processes [9]. The healing of a thermoplastic interface occurs as chain ends diffuse across the boundary and entangle with the adjacent polymer $[10,11,12]$. The mechanical strength of the interface, $\sigma$, increases with time in contact, $t$, until the interface achieves the strength of the fully healed composite interface $\sigma_{\infty}$. This ratio is defined as the degree of healing, $D_{h}$.

$$
D_{h}=\frac{\sigma_{w}}{\sigma_{\infty}}=\left(\frac{G_{1 c, w}}{G_{1 c, \infty}}\right)^{1 / 2}=\left(\frac{t_{w}}{t_{w, \infty}}\right)^{1 / 4}
$$

Here, $w$, denotes that this is a welding process, and $t_{w, \infty}$ is the welding time $\left(t_{w}\right)$ required to complete the welding process, such that the interface strength $\left(\sigma_{w}\right)$ reaches the strength of the bulk polymer $\left(\sigma_{\infty}\right)$. Alternatively, $D_{h}$ can be expressed in terms of the weld fracture energy release rate, $G_{1 c, w}$, relative to that of the bulk polymer, $G_{1 c, \infty}$. Eq. (1) has been verified many times for different polymer of varying molecular weight $[10,11,12]$. In isothermal conditions, the welding time, $t_{w, \infty}$, of a monodisperse linear polymer of given 
molecular weight may be modelled by an Arrhenius law:

$$
t_{w, \infty}=A \exp \left(\frac{E_{a}}{R T}\right)
$$

From Eq.(1) the welding time is classically considered to be identical to the reptation time of the polymer, $t_{R}=t_{w, \infty}[10,13]$. The reptation time is intimately linked to the viscoelastic nature of the polymer: it depends on the chemistry, molecular structure, molecular weight, and temperature. The most reliable method to characterize $t_{R}$ is a stress-relaxation test. This can be performed on a displacement controlled rheometer. With the polymer melt situated between the plates (cone or parallel) and in an equilibrium relaxed state, a known shear strain is abruptly applied. Locked into the fix shear state, the shear stress in the polymer decays over time as the polymer relaxes. From this test the relaxation time, $\lambda_{R}$, is determined from the Maxwell model for a viscoelastic fluid,

$$
\lambda_{R}=\frac{\eta_{0}}{G_{0}^{N}}
$$

where $\eta_{0}$ is the zero shear viscosity (of the newtonian plateau) and $G_{0}^{N}$ is the plateau shear modulus [14]. This type of test, as long as it stays within the linear viscoelastic regime, can be very characteristic of the polymer reptation time. In the initial sheared state, polymer chains must disentangle from their strained configuration and gradually reach a state of no stress, as they complete a full reptation process. As such, we can infer that the relaxation time is equivalent to the polymer reptation time $[13,14]$.

Complex fluids like polymer melts are often not well characterized by a single relaxation time. The visco-elastic response can be fitted with a series of relaxation modes

$$
G(t)=\sum G_{i} \exp \left(-t / \lambda_{i}\right)
$$

where $G_{i}$ and $\lambda_{i}$ are a discrete set of moduli and relaxation times which capture the rheological response. If the set of discrete parameter pairs is small (4 or fewer), a physical meaning can more easily be assigned to each. Conversely, with an infinite set, a continuous relaxation spectrum can be used to transform all linear visco-elastic functions between each other (relaxation modulus, 
creep-compliance, complex modulus/compliance) [15]. This allows a variety of rheological protocols to capture the same behavior on different time scales. From the discrete spectrum, the longest relaxation time is said to be equivalent to the interface healing time [16].

In order to directly assess the degree of healing in a welded joint, samples must be welded under controlled conditions (pressure, temperature, and time) and then tested mechanically to observe the interface strength. Lee and Springer [6] proposed to test samples bonded under a press under mode I tensile loading. Their study was focused on autoclave processing of laminates, rather than rapid welding processes. Lamethe et al. [17] proposed a methodology to measure the fracture energy of samples welded under controlled nonisothermal conditions. In a previous work, Avenet et al. [18], developed an apparatus (TACOMA bench) for welding full double cantilever beam specimens isothermally with residence times as short as 5 seconds by employing rapid cooling. The methodology was very effective at determining the welding time in Eq. (2) through a series of tests (varying temperature and time) fit to Eq. 1.

Historically, welding times for commercially available aerospace grad PEEK and PEKK polymers have been shown be on the order of $10^{3} \mathrm{~s} \quad[6,19,20]$; however, some currently available PAEK polymers are better tuned for welding processes with residence times of the order of a few seconds. Choupin et al. [21] demonstrated that polymer degradation is a major challenge when processing PEKK (or PEEK) composites: above the melt temperature $\left(340^{\circ} \mathrm{C}\right)$ the polymer backbone can break and initiate crosslinking; thus, limiting the ability to crystallize and increasing the polymer viscosity. An exponential increase in viscosity over time and temperature (in an Nitrogen atmosphere) showed that crosslinkining was a temperature driven kinetic process [22]. They confirmed an increase in molecular weight with Gel Permeation Chromatography (GPC).

After presenting the PEKK 7002 material used in this study, section 3 presents the procedure to identify the longest relaxation time of the polymer. In section 4 the mechanical welding time is characterized with the existing TACOMA welding bench and a double cantilever beam test. Finally, the corre- 
lation between these two approaches is discussed in the last section. Thermal history is also shown to impact the weldability.

\section{Materials}

In this study, we characterize PEKK 7002 produced by Arkema. The 7000 series designates that the polymer has a ratio of 70:30 of T:I (Terephthaloyl to Isophthaloyl) and the final digit specifies the melt volume flow index. The PEKK 7002 has a rather low index and thus a high viscosity and high mechanical properties. On the contrary, its processability is limited.

\subsection{Rheological samples}

For rheological characterization, flat discs of diameter $25 \mathrm{~mm}$ and thickness $2 \mathrm{~mm}$ were manufactured from neat PEKK pellets. Pellets were placed inside of a mold at $350^{\circ} \mathrm{C}$ for 5 minutes (until appearing amorphous) and then compression molded at 20 bars. Subsequently, while under pressure, the mold was cooled with water; however, the samples were able to crystallize. The crystallinity was not important at this stage because once in the rheometer, the samples were certain to melt. The importance of this process is that the cycle time was short, so that degradation would be minimized, and samples had no air bubbles when placed in the rheometer.

\subsection{Adhesion samples}

Carbon fiber composite plates were manufactured with a $[0,90]_{3 s}$ manual layup using $194 \mathrm{~g} / \mathrm{m}^{2}$ carbon-PEKK prepreg from Toho Tenax. On one of the surface, a $50 \mu \mathrm{m}$ thick film of neat PEKK 7002 polymer was applied to ease the welding process. The plates were then consolidated in an autoclave according to the cycle shown in Fig. 1 where the sample was kept above $350^{\circ} \mathrm{C}$ for 90 minutes. Note that a typical autoclave cycle for thermoplastic composite consolidation include a 30 minute dwell at the peak temperature; however, in this study the cycle was adapted from a standard thermoset procedure and has a longer dwell than the one recommended by the manufacturer. 
The $3.2 \mathrm{~mm}$ thick composites plates were cut using a Protomax waterjet cutting machine to the sample specification of $25 \mathrm{~mm}$ by $125 \mathrm{~mm}$ (cf Figure 7 ) where the surface ply orientation is along the length direction. A countersunk hole was then machined on the matrix-rich surface, $10 \mathrm{~mm}$ from one end, to insert a homemade loading pin used for gripping the sample during the mechanical test [18]. After a manual cleaning of the surfaces with acetone, the samples were placed into a vacuum chamber at $180^{\circ} \mathrm{C}$ for 20 hours to remove the residual water contained into the samples and limit deconsolidation during the heating phase of the welding process.

The subsequent controlled welding process is described later, in section 4.1.

\subsection{Thermal history effect}

Concurrently, in order to quantify the effect of thermal degradation on the welding ability of the composite samples, aging tests were conducted on the already autoclave-consolidated composite plates. To do that, an autoclave composite plate was vacuum-bagged in an oven and heated up to $380^{\circ} \mathrm{C}$ with an isothermal step of 2 hours. Vacuum bagging was chosen to prevent deconsolidation during the thermal cycle and temperature was recorded with a thermocouple placed on the top of the plate during the whole aging process (Fig. 1). The aged composite plates were then cut and dried according to the experimental protocol previously described, before welding stage.

\section{Rheological time identification}

To characterize the rheological behavior of PEKK in the molten state, a Thermofischer HAAKE MARS III rheometer was used. The temperature controlled chamber (CTC) configuration was used to maintain the sample isothermally at elevated temperature $\left(350^{\circ} \mathrm{C}<\mathrm{T}<420^{\circ} \mathrm{C}\right)$ throughout testing. A nitrogen purge was also maintained throughout testing to negate the potential for oxidation. Tests were conducted with $35 \mathrm{~mm}$ aluminum parallel plates with a gap of $1 \mathrm{~mm}$. 


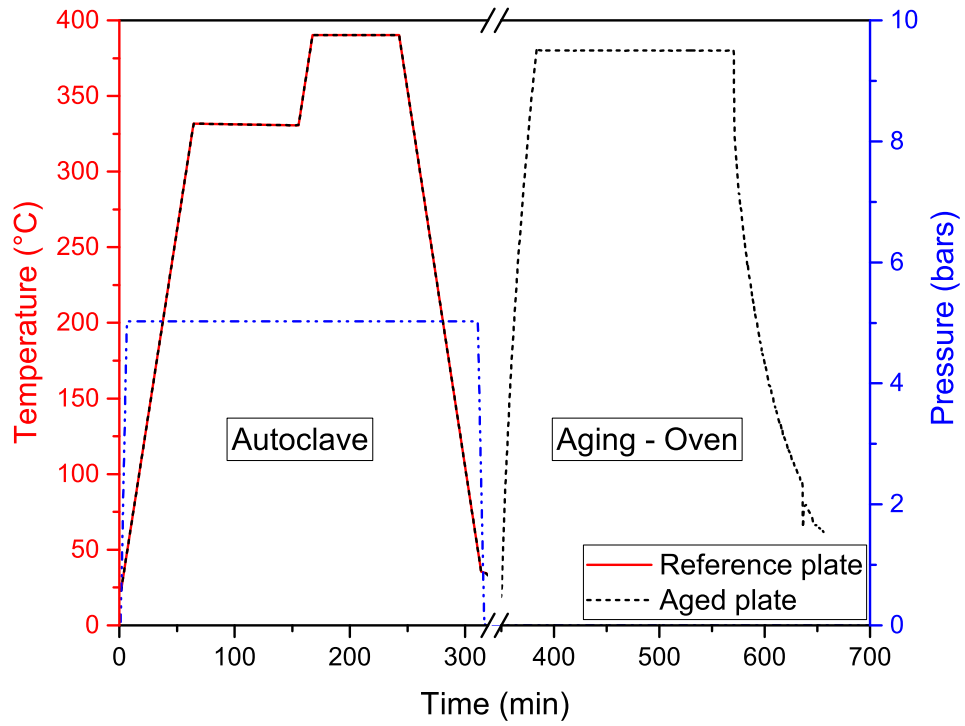

Figure 1: Pre-processing cycles used to produce composite laminates. The reference plate is manufactured with a five hour autoclave cycle which includes a 90 min dwell at $330^{\circ} \mathrm{C}$ followed by a $80 \mathrm{~min}$ dwell at $380^{\circ} \mathrm{C}$. Samples selected to undergo further thermal degradation were post-processed in and oven vacuum-bag for $2 \mathrm{hrs}$ at $380^{\circ} \mathrm{C}$. 
To prepare the test, the rheometer was calibrated at room temperature. Without the sample present, the rheometer was preheated to the test temperature. Once the chamber temperature had stabilized, the $25 \mathrm{~mm}$ samples was placed on the $35 \mathrm{~mm}$ plate and the chamber was closed. The gap was adjusted to $1 \mathrm{~mm}$, where the polymer would wet to the edges of the plates.

\subsection{Oscillatory tests}

It is known that PEKK polymers are not stable above their melting temperature. This is characterized by the increase of viscosity over time [21] that could be due to crosslinking of the polymer macromolecules.

The effect of temperature on the rheological properties of the polymer was assessed by means of oscillatory rheological tests at constant temperature at a frequency of $1 \mathrm{rad} / \mathrm{s}$ with $1 \%$ strain. The test was repeated for 4 temperatures: $360,380,400$ and $420^{\circ} \mathrm{C}$. The temperatures were chosen to be higher than the melting temperature of the materials and are characteristic of most of the industrial processes. Autoclave manufacturing for example is characterized by long residence times at high temperature which could induce macromolecular changes.

Figure 2 shows an important increase in the storage modulus at high temperature which could be attributed to a decrease in chain mobility and confirms crosslinking of the materials. This crosslinking seems also to be faster at higher temperatures validating that this is a thermo-dependent phenomenon as already demonstrated for PEKK [21] and PEEK [23]. As both the storage and loss moduli increase with dwell time, the intersection between $G^{\prime}$ and $G^{\prime \prime}$ takes place at lower frequencies and the relaxation times are consequently higher.

\subsection{Creep recovery tests}

Oscillatory shear sweep tests were performed in the high frequency (pulsations $0.1 \mathrm{rad} / \mathrm{s} \leq \omega \leq 100 \mathrm{rad} / \mathrm{s}$ ) domain to get the evolution of $G^{\prime}$ and $G^{\prime \prime}$ and obtain the longest relaxation time of the polymer. 

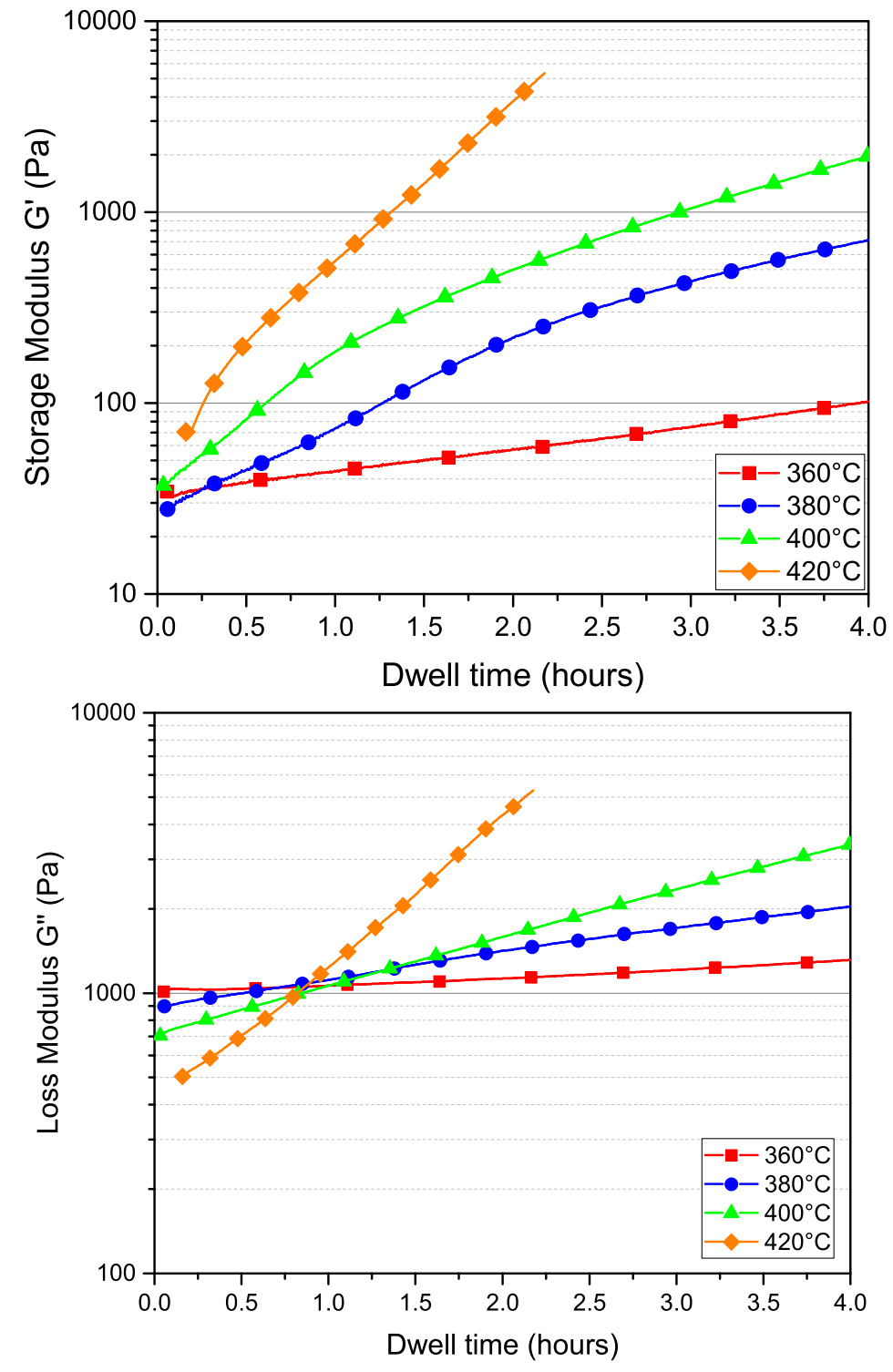

Figure 2: Evolution of the storage modulus and loss modulus with dwell time under a frequency of $1 \mathrm{rad} / \mathrm{s}$ with $1 \%$ strain at constant temperatures. 
With the primary goal of the rheological characterization being the calculation of the lowest relaxation time, we aimed to observe the storage and loss moduli $G^{\prime} \& G^{\prime \prime}$ over a wide range of frequencies. Namely, it was important to measure the low frequencies, at the newtownian plateau. In the domain of high frequency (pulsations $0.1 \leq \omega \leq 100$ ), $G^{\prime} \& G^{\prime \prime}$ can be quickly measured (within a couple minutes) with an oscillatory shear sweep. The problem to contend with is that the low frequencies take a long time to acquire, and during that time, the polymer is crosslinking. To obtain this data in a more timely fashion, a creep-recovery method was used to characterize the visco-elastic response at low frequencies (i.e. low shear rate and/or long relaxation times).

The experimental procedure starts with a frequency sweep, with $1 \%$ strain. Beginning at $100 \mathrm{rad} / \mathrm{s}$, the frequency is stepped down logarthimically by 5 frequencies per decade and ending at $0.1 \mathrm{rad} / \mathrm{s}$. The end point $0.1 \mathrm{rad} / \mathrm{s}$ was chosen for consideration of the acquisition time: each additional frequency takes exponentially longer to complete. In total, the frequency sweep takes a total of $12 \mathrm{~min}$ to complete. At the end of the frequency sweep, the material is allowed to recover for $3 \mathrm{~min}$ to ensure there is little residual stress from the previous oscillatory test. Once the samples nearly stops deforming $\left(\dot{\gamma}<0.001 \mathrm{~s}^{-1}\right)$, the creep-recovery test is initiated. In the creep-recovery test, the applied stress must be set such that the observed shear rate is low enough to ensure the material is within the linear elastic regime, but not too low for the acquisition of the rheometer. Initial tests showed that an applied stress of $5 \mathrm{~Pa}$ to $30 \mathrm{~Pa}$ is adequate, depending on the state of crosslinking. Additionally, the duration of the applied stress, $t=t_{2}$, must be sufficiently long to ensure the material is deformed past its elastic limit, to the point where the deformation is only viscous $\left(t>t_{1}\right)$. This time corresponds roughly to the relaxation time of the material. Initially the deformation time can be short (300 s), but as the material crosslinks, longer deformation time is necessary, (1800 s). After the creep test, the material is allowed at least twice as long (at zero stress) to recover from residual stress caused by the previous oscillatory test. At the end of the creeprecovery test, the sequence is repeated, beginning again with the frequency 


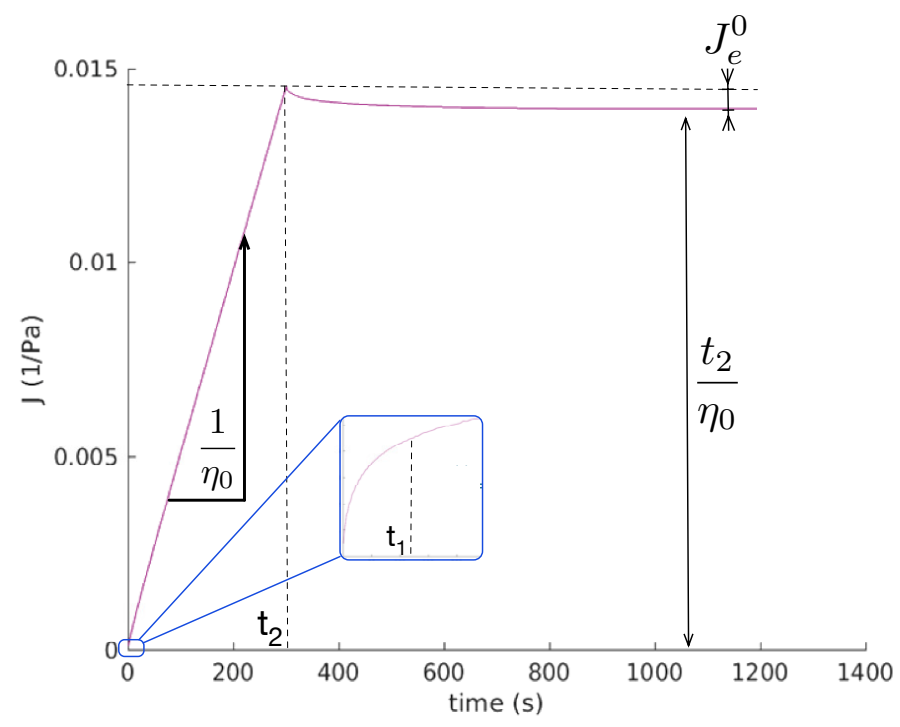

Figure 3: Creep compliance versus time obtained with the creep recovery test for $100 \mathrm{~Pa}$ at $380^{\circ} \mathrm{C}$. The close-up shows that the viscous regime is reached quickly in this case.

sweep.

The creep compliance

$$
J(t)=\frac{\gamma(t)}{\sigma_{0}}=J_{e}(t)+\frac{t}{\eta_{0}}
$$

is the sum of the elastic deformation (recoverable) and the viscous deformation (non-recoverable). In Figure 3 an annotated creep-recovery curve shows how material constants $\left(\eta_{0}\right.$ and $\left.J_{e}^{0}\right)$ are measured. After some time $\left(t=t_{1}\right)$, the shear rate becomes constant and the elastic deformation reaches a plateau which defines the compliance limit, $J_{e}^{0}$. The compliance limit, $J_{e}^{0}$, was taken from the recovery period since a long recovery period was required in the test protocol to ensure the polymer was in a quiescent state before the next iteration.

A Fourier transform is able to convert the creep compliance from the time domain to a frequency domain and to obtain the storage compliance $J^{\prime}[15]$ and the loss compliance $J^{\prime \prime}$

$$
J^{\prime}(\omega)=J_{e}^{0}-\omega \int_{0}^{\infty}\left[J_{e}^{0}-J(t)+\frac{t}{\eta_{0}}\right] \sin (\omega t) d t
$$




$$
J^{\prime \prime}(\omega)=\frac{1}{\omega \eta_{0}}+\omega \int_{0}^{\infty}\left[J_{e}^{0}-J(t)+\frac{t}{\eta_{0}}\right] \cos (\omega t) d t
$$

Practically, the integration was performed over the duration of the creep period of the test $\left(0 \leq t \leq t_{2}\right)$. The storage and loss compliance can be simply converted to their respective moduli by the relations:

$$
G^{\prime}=\frac{J^{\prime}}{J^{\prime 2}+J^{\prime \prime 2}}
$$

and

$$
G^{\prime \prime}=\frac{J^{\prime \prime}}{J^{\prime 2}+J^{\prime \prime 2}}
$$

Figure 4 shows the evolution of the storage and loss moduli over frequency. The lines correspond to the data acquired with the classical oscillatory rheology whereas the dotted lines are constructed from the Fourier transform of the creep recovery tests in order to achieve lower frequencies. The Figure shows that with time increasing, the $G^{\prime}$ and $G^{\prime \prime}$ curves are shifting to the higher values indicating a network change attributed to crosslinking.

In the limit of very low frequencies $G^{\prime}=J_{e}^{0} \eta_{0}^{2} \omega^{2}$ and $G^{\prime \prime}=\omega \eta_{0}$. Therefore, a good approximation for the longest relaxation time would be at the intersection $G^{\prime}=G^{\prime \prime}$. Using the Cox-Merz rule that $\omega \approx \dot{\gamma} \approx 1 / \lambda$, we can see that

$$
\lambda_{R}=J_{e}^{0} \eta_{0}
$$

Thus, the longest relaxation times were finally determined from the creep recovery tests and were calculated as the product of the elastic compliance limit and the newtonian plateau viscosity (Eq.10). The procedure was repeated over time at constant temperature to observe the changes in relaxation times induced by thermal history.

\section{Mechanical time identification}

In order to correlate the rheological times characterized in the previous section with characteristic mechanical time related to the healing phenomenon, a welding campaign is conducted. 

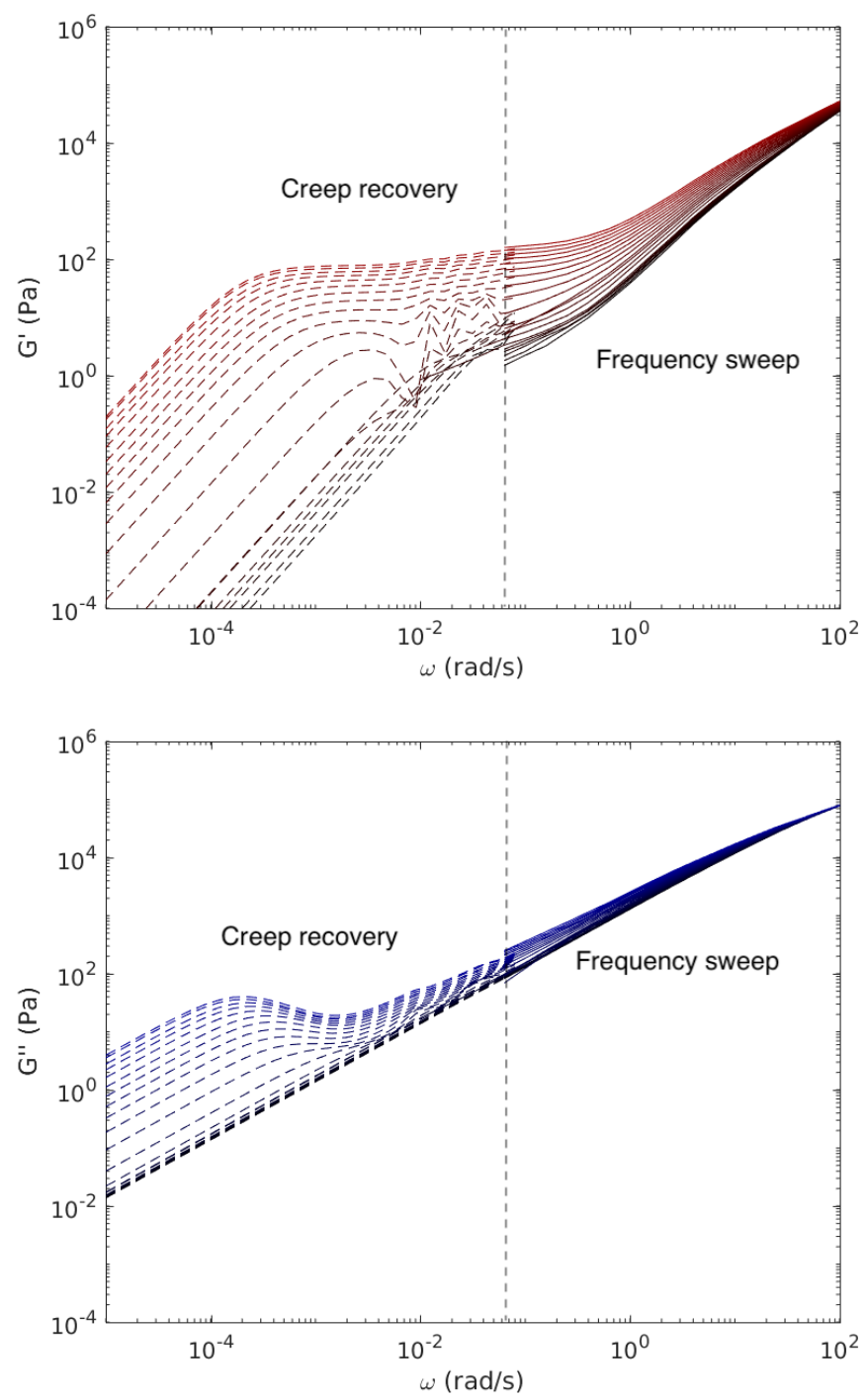

Figure 4: Storage and loss moduli versus frequency for PEKK 7002 in $\mathrm{N}_{2}$ gas at $350^{\circ} \mathrm{C}$. The high frequency range (short time response) is captured with an oscillatory frequency sweep, whereas the low frequency range (long time response) is measured by a creep-Recovery method and converted to frequency space with a Fourier transform. Iterating the protocol sequence shows how the visco-elastic response changes as the polymer degrades over time. Each repetition shows a longer relaxation time. 


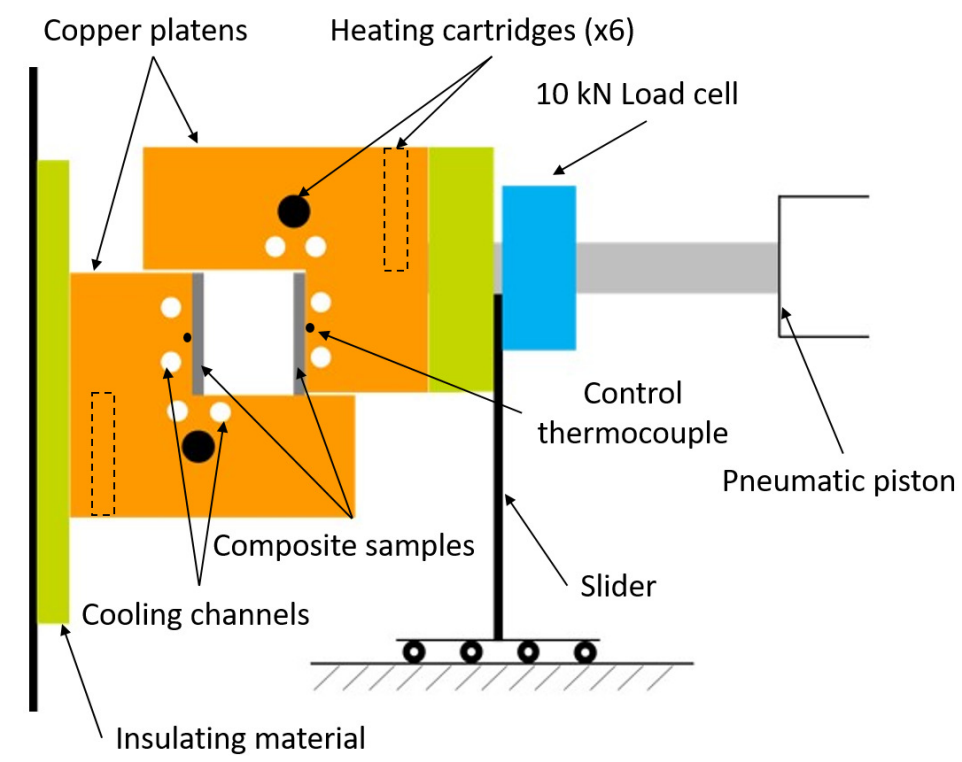

Figure 5: Principle of the experimental TACOMA setup, designed for welding under isothermal conditions. Welding process is stopped thanks to a fast water cooling.

\subsection{Welding procedure}

Welding experiments were carried out on an experimental welding bench (TACOMA), presented in a previous article [18] and shown in Fig. 5, under constant isothermal conditions.

This experimental welding bench was designed to enable quenching of the composite sample and thus to perform stopped welding experiments. Thermocouples were embedded $1 \mathrm{~mm}$ from the sample surface in the copper mold to control and monitor the temperature and the heating was performed thanks to heating cartridges. Thermal homogeneity is ensured since all the samples are heated and pressed together in a confined mold. Furthermore, two channels were machined in each platen near the sample surface for water cooling of the setup. The pressure was applied by means of a pneumatic piston attached in series to the moving platen and the contact force was recorded by a $10 \mathrm{kN}$ load cell.

Prior to welding experiments, the coupons were covered with polyimide tape 


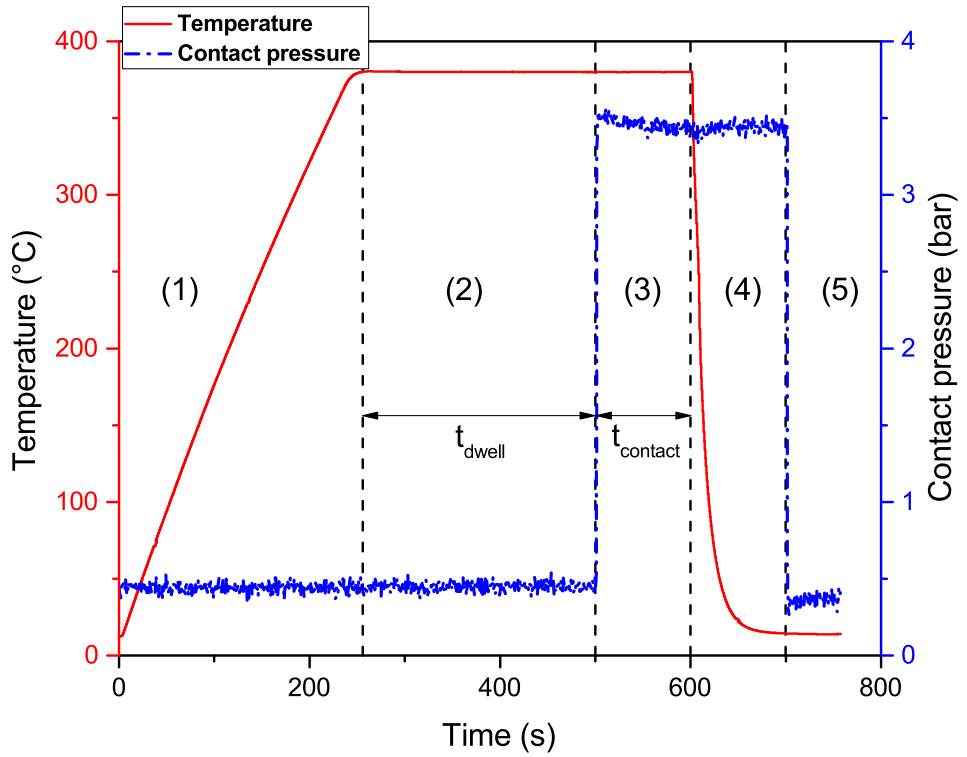

Figure 6: Experimental welding procedure. The heating (1) and temperature stabilization (2) occur before the samples are put in contact. The welding time is $t_{\text {contact }}(3)$ and quenching occurs in phase (4). 
to facilitate removal after the welding sequence. First, the two composite samples were placed in the setup, resting on their sides and the matrix-rich surfaces were facing each other but they were not in contact. A $50 \mathrm{~mm}$ long non-adhesive aluminum foil was also placed between the coupons as shown in Fig. 7. This allowed the creation of an initiation crack for fracture test. As shown in Fig. 6, the device is heated from room temperature up to a temperature higher than the melting temperature of the polymer matrix (1) until homogenization of the temperature inside the mold and at the interface of the coupons (2). Once the setpoint temperature is reached and homogeneous inside the mold, contact is initiated between the coupons with the use of the pneumatic piston to weld the samples during a defined contact time $t_{c}(3)$. Welding process is rapidly stopped thanks to the fast cooling of the setup with water circulation (4) and finally the pressure is released (5).

\subsection{Double cantilever beam fracture test}

Mechanical quality of the welded interfaces was assessed by mode I double cantilever beam fracture test according to ASTM standard. Samples were mounted on a $100 \mathrm{kN}$ Zwick Roell tensile machine thanks to the loading pins and homemade gripping system. Tests were conducted in quasi-static condition at a fixed displacement rate of $1 \mathrm{~mm} \cdot \mathrm{min}^{-1}$. During the test, the displacement and force were continuously recorded. The welded samples were not cut after welding so that the dimensions of the Double Cantilever Beam samples are also $25 \mathrm{~mm}$ by $125 \mathrm{~mm}$.

The load-displacement curves from the double cantilever beam tests were characterized by a strong unstable crack propagation [18] probably caused by a non-uniformity of the matrix-rich layer at the interface [24]. Interlaminar fracture energy release rate

$$
G_{1 c}=\frac{3 P \delta F}{2 b a_{0}}
$$

was then calculated at the initiation of the crack, corresponding to the first drop

of load. In the above, $P$ is the peak load, $\delta$ the displacement, $b$ the width of the sample, $a_{0}$ the initial crack length which is the length of the non-adhesive 

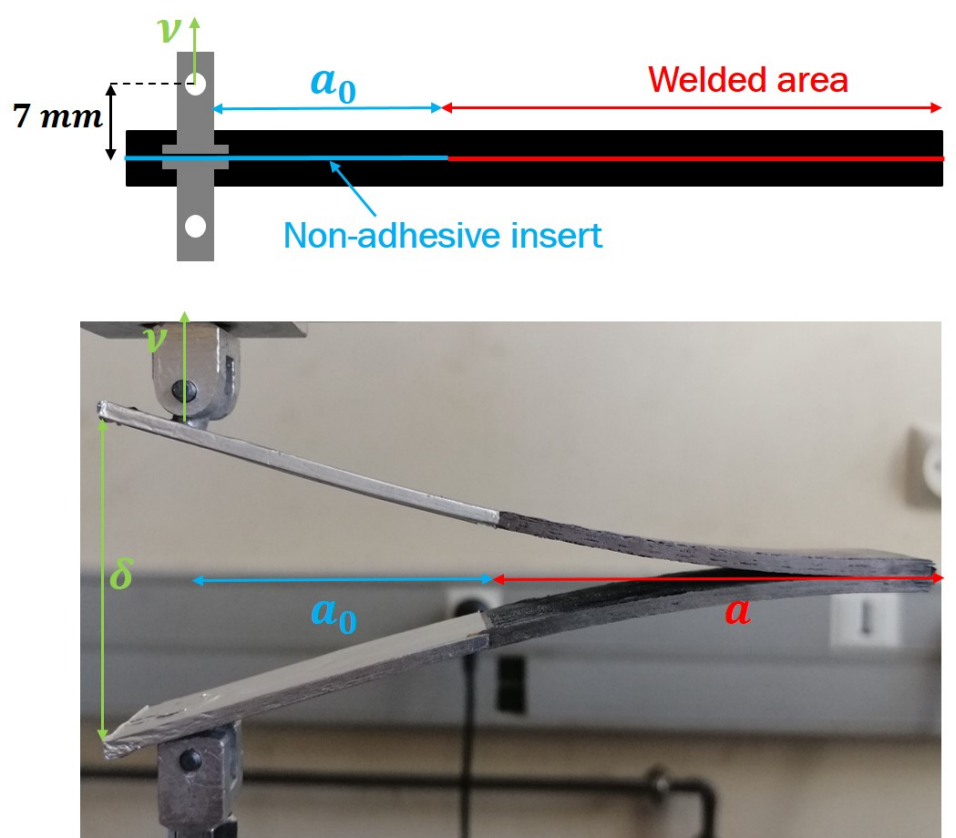

Figure 7: Double Cantilever Beam (DCB) fracture test set-up. $a_{0}$ is the initiation crack length.

inserted aluminum foil and $F$ is a correction factor accounting for large displacement effects, i.e. shortening of the moment arm and tilting of the loading pins. It has previously been demonstrated that this value is representative of the overall adhesion of the assembly [18].

\subsection{Analysis}

The welding time was determined experimentally for three isothermal temperatures $\left(355,370\right.$ and $\left.380^{\circ} \mathrm{C}\right)$ by means of welding tests performed using the TACOMA setup for different residence times. Adhesion energy was determined from load-displacement in DCB fracture tests with Eq. 11. Degree of healing at the crack initiation was evaluated via Eq. 1, where $G_{1 c}$ is evaluated for each sample of corresponding welding time. $G 1 c, \infty$ was determined to be 2.3 $\mathrm{kJ} . \mathrm{m}^{-} 2$ from a stable maximum plateau where additional welding time results in no improvement in interface strength-corresponding to the fracture energy of 


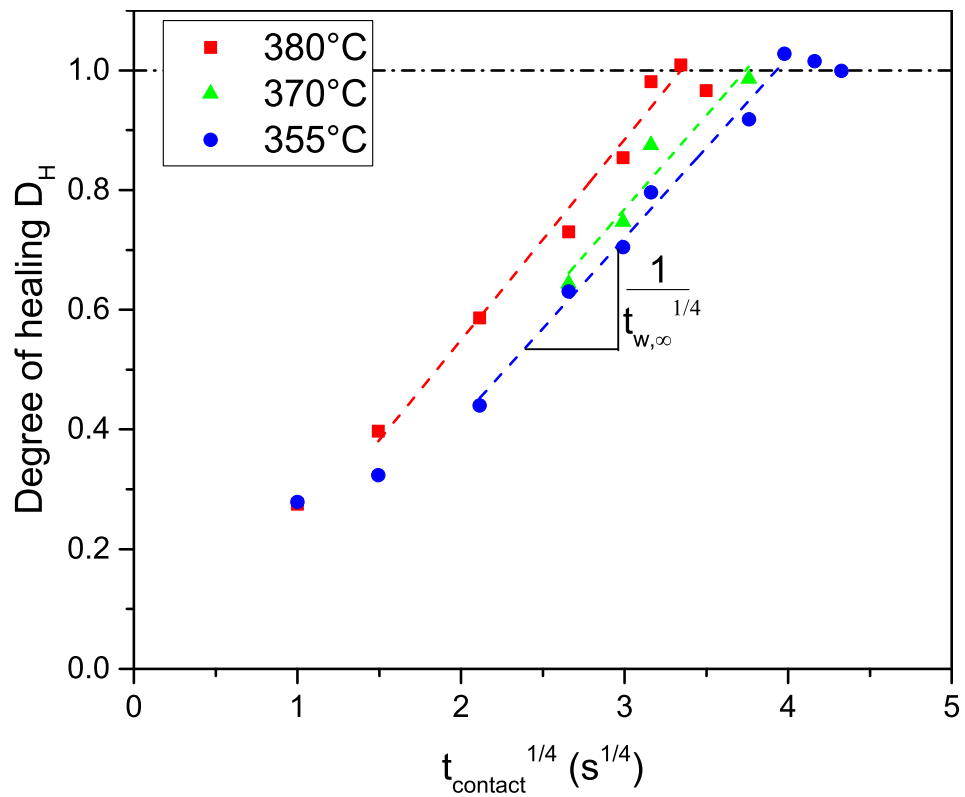

Figure 8: Degree of healing at the crack initiation versus the fourth root of contact time for three isothermal temperatures. Welding time can directly be obtained from the slope of the linear fit [18].

\begin{tabular}{lccc}
\hline Temperature $T\left({ }^{c} \mathrm{C}\right)$ & 355 & 370 & 380 \\
Mechanical welding time $t_{w, \infty}(\mathrm{s})$ & 121 & 104 & 79 \\
\hline
\end{tabular}

Table 1: Mechanical welding times characterized at different isothermal temperatures

the fully healed interface. This high value compared to other high performance composites but it has been related to the amorphous state of the interface [18].

Degree of healing was then plotted against the fourth root of contact time for the three isothermal temperatures (see Figure 8). In this case, the first data points were shown to be not associated to pure healing mechanism but also to the establishment of intimate contact. Therefore, these points were discarded and the welding times were directly taken from the slope of the linear fit. They are given in Table 1. Finally they were plotted against the inverse of temperature to identify the corresponding Arrhenius relationship given in Eq. (2), where 


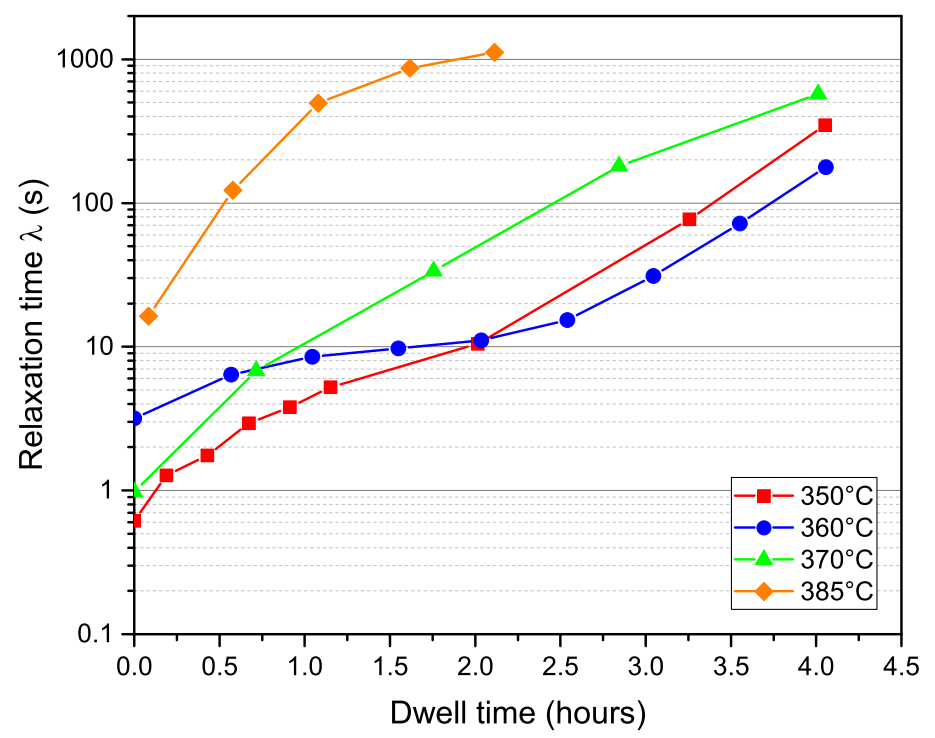

Figure 9: Evolution of the longest relaxation time of PEKK 7002 versus dwell time. Relaxation time increases with material evolution. This indicates that the pre-processing of the material will subsequently affect the welding process.

the parameters $\mathrm{E}_{a}=55,5 \mathrm{~kJ} \cdot \mathrm{mol}^{-1}$ and $\mathrm{A}=3,01 \cdot 10^{-3} \mathrm{~s}$ are respectively the activation energy and the identified pre-exponential factor.

\section{Discussion}

\subsection{Rheological test as a mean to quantify adhesion}

From the rheological characterization it was possible to predict the welding time of the composite based on its thermal history. The relaxation times are plotted for each isothermal temperatures against dwell time in Fig. 9. It shows that for a fresh polymer matrix, i.e. for $t=0$, the relaxation time is of about 1 second. This corresponds to the data obtained in the literature $[17,16]$. As preprocessing time increases, the relaxation time increases consequently and can even reach values of a few thousand seconds as it was announced in $[6,19,20]$.

Adhesion measurements on the composite at different temperatures allowed the identification of the healing kinetics and the associated welding time. It 
should be recalled that the composite plate has originally been processed at $380^{\circ} \mathrm{C}$ for one hour $\left(90\right.$ minutes above $350^{\circ} \mathrm{C}$ ) in autoclave before welding experiments. Although this is not the cycle recommended by the manufacturer, the adhesion data is characteristic of these aging conditions. Concurrently, the rheological protocol aimed at replicating the pre-processing of the composite and characterizing the corresponding relaxation time.

Figure 9 presents, the evolution of relaxation time at $385^{\circ} \mathrm{C}$. This corresponds to the data acquired after in-situ crosslinking of the polymer matrix in the rheometer at this temperature of $385^{\circ} \mathrm{C}$ and over a wide range of dwell times. If one considers the processing conditions previously recalled for the PEKK-Carbon composite plate of this study, it is possible to estimate the analogous relaxation time and the welding time.

Thus, for a dwell temperature of $380^{\circ} \mathrm{C}$ and dwell time of 3600 seconds the value of the welding time for this composite plate is of a hundred of seconds.

In parallel, the adhesion measurements from the welding experiments showed that the time for a full healing of the interface is also of a hundred seconds depending on the welding temperature (see Table 1). This comparison between rheological and adhesion characterization finally showed a good correlation for a given process condition which is the autoclave pre-processing dwell in this case and proved that rheological characterization of the polymer matrix can thereby be a mean to quantify adhesion time in the case of composite material.

Such procedure is much more rapid since it only takes about one day to gather the data from rheological measurements using a commercial rheometer. On the contrary, adhesion measurements are characterization campaigns that may last several weeks to perform. Moreover, the welding tests and the associated mechanical characterization require specific apparatus. Finally, adhesion measurements require a significant number of samples, obtained from preconsolidated laminates for the identification of the healing kinetics; when rheological characterization only necessitates polymer pellets which is thus much less expensive. 


\subsection{Effect of Thermal History}

To confirm the effect of thermal history, welding tests at $380^{\circ} \mathrm{C}$ were performed on the composite plates aged for 2 hours at $380^{\circ} \mathrm{C}$ as described in section 2.3 using the TACOMA set-up according to the experimental protocol described in section 4.1. The force-displacement curves from the corresponding DCB tests are shown in Fig.10 and compared with the force-displacement curves from the reference autoclaved composite plate. First, the initial slopes are the same for the two plates indicating that the elastic performances at room temperature of the composite coupons after thermal aging did not change. Then, micrograph analysis of the aged plate also showed no apparent defects. These validate that no deconsolidation occurred during the aging process. Thereby, the observed changes are solely related to the quality of the welded joint.

For the aged composite plates, only a few adhesion tests were successful and led to few data points. Indeed, such thermal aging was shown to be the lower limit for adhesion development and for most of the samples no adhesion could be obtained even for very long contact time. This is most probably related to a high degree of thermal degradation and thus higher crosslinking of the material.

Considering that the original composite plates were consolidated in autoclave for about 90 minutes at high temperature, the total dwell time of the aged plates is then of about 3.5 hours. From the experimental evolution of the relaxation time of the matrix previously presented, at such dwell times, the relaxation time and therefore the welding time is expected to be extremely long, of about one hour.

The corresponding initiation values of the interlaminar fracture energy are then plotted in Figure 10 against the square root of contact time for both the original and the aged composite plates. For the same welding temperature and contact time, the interlaminar fracture energy value for the aged composite is an order of magnitude lower than the reference composite plate. The thermal aging has affected the weldability of the material by hindering the chain diffusion at the interface. It is believed that the macromolecular network has branched and that it takes longer for the chains to move and cross the interface. 

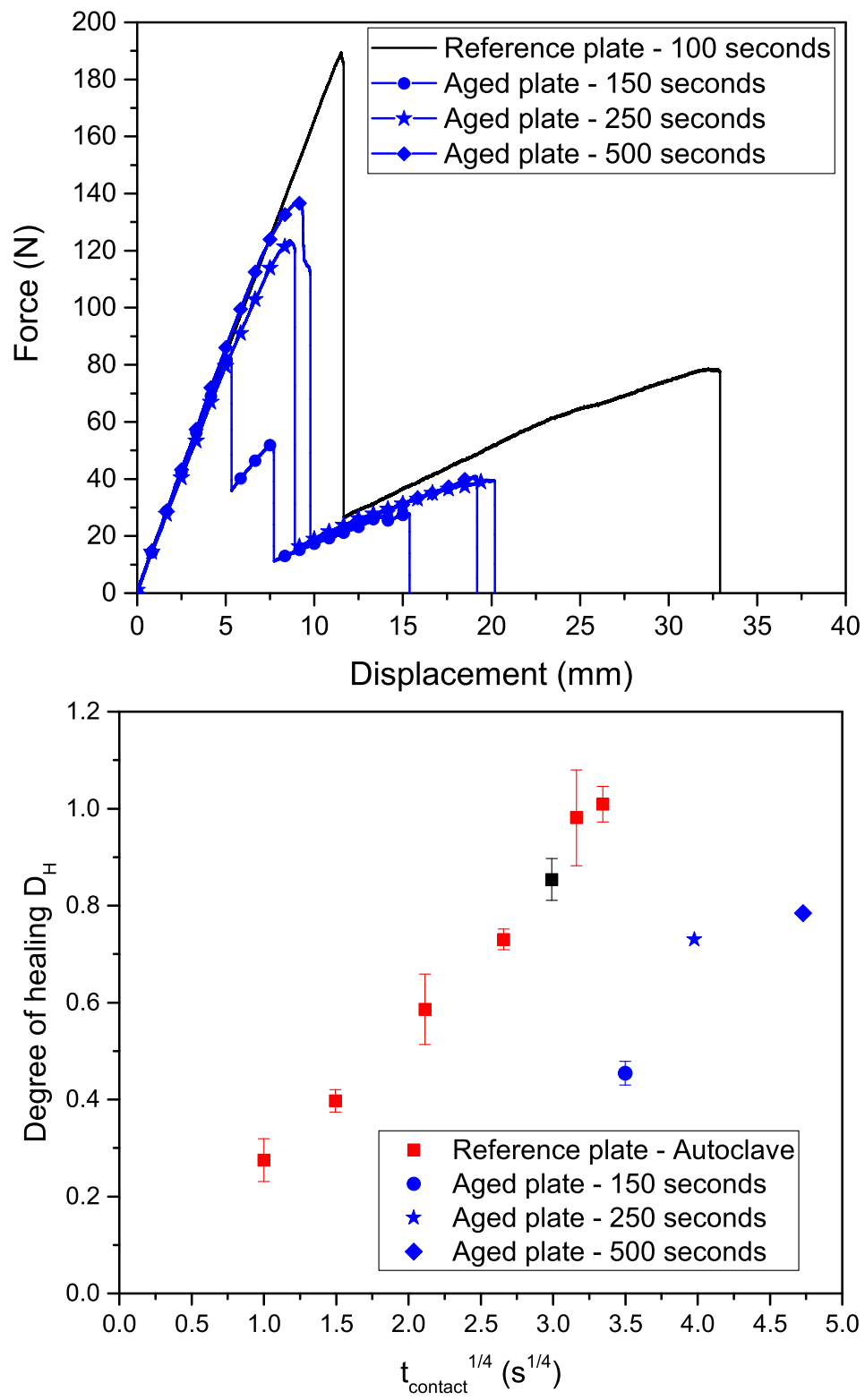

Figure 10: Comparison between the reference autoclaved plate and the aged plate at $380^{\circ} \mathrm{C}$ for 2 hours. Coupons were welded at $380^{\circ} \mathrm{C}$ with different contact times. (a) Force-displacement curves and (b) degree of healing. Even at processing temperature, the additionally dwell drastically reduces the welding potential of the carbon PEKK system 
As a result, the pre-processing will affect the welding process. For example, in the case of our composite material, the plates were consolidated in autoclave at $380^{\circ} \mathrm{C}$ for at least 1 hour. Both rheological and mechanical approaches give an estimate of a hundred seconds for the welding time, but more severe thermal history hinder adhesion development. Continuous welding processes such as automated fibre placement which make use of fresher composite substrates like prepreg tapes are expected to gain full mechanical adhesion for much shorter processing time. But the thermal history experienced by high performance thermoplastic composite coupons could then be a major limiting step in the development of adhesion.

\section{Conclusions}

In this work, the welding kinetics of a high performance thermoplastic composite was characterized. The study is twofold. First a rheological procedure gave the longest relaxation time of the polymer. A creep recovery method enabled to overcome the polymer degradation issue. Then a mechanical study of coupons welded using the existing TACOMA bench gave the mechanical healing times. Mechanical tests were also performed with an over-aged composite plate.

The main contributions of the work are the correlations between the matrix rheological times and the composite mechanical bonding times. Additionally, PEKK 7002 was shown to evolve with time at high temperature. This is attributed to crosslinking and impacts the relaxation time. This effect is consistently observed on the healing time identified with mechanical tests. Thus, polymer degradation during the pre-processing step, such as autoclave cycle for instance, influences the subsequent weldability of the structures. As with every polymer processing scheme, the thermal history should be managed in order to minimize polymer degradation. The specimens used in this study reported welding times on the order of 100 seconds but the autoclave cycle included a 90 minute dwell period, which is longer than recommend. It is shown here that a shorter autoclave cycle will produce more weldable samples. 


\section{Acknowledgements}

The authors would like to acknowledge the funding they received from the PERFORM program managed by IRT Jules Verne (French Institute for Research and Technology in Advanced Manufacturing Technologies for Composite, Metallic and Hybrid Structures). The authors wish to associate the industrial partners of the PERFORM project: Airbus, Stelia Aerospace, Faurecia, Naval Group and Daher. Part of this study was also funded by the SIDEFFECT project managed by IRT Jules Verne. The industrial and academic partners of this project are respectively Stelia Aerospace, Airbus, Europe Technologies, Hutchinson, Cetim, Aviacomp, and IREENA Université de Nantes.

The authors would like to thank Professor Erwan Verron for his fruitful discussion about fracture mechanics. Juliana Amirdine also helped with the rheology analysis. Nicolas Boyard provided useful help and insights on the aging effect. Finally, the bench design and manufacturing would not have been possible without the help of Nicolas Lefevre and Jérôme Delmas.

[1] Y. Yan, W.-D. Wen, F.-K. Chang, and P. Shyprykevich. Experimental study on clamping effects on the tensile strength of composite plates with a bolt-filled hole. Composites Part A: Applied Science and Manufacturing, 30(10):1215 - 1229, 1999.

[2] C. Ageorges, L. Ye, and M. Hou. Advances in fusion bonding techniques for joining thermoplastic matrix composites: A review. Composites - Part A: Applied Science and Manufacturing, 32(6):839-857, 2001.

[3] C.T. Sun, Islup Chung, and I.Y. Chang. Modeling of elastic-plastic behavior of $\mathrm{ldf}^{\mathrm{TM}}$ and continuous fiber reinforced as-4/pekk composites. Composites Science and Technology, 43(4):339 - 345, 1992.

[4] T.E. Attwood, P.C. Dawson, J.L. Freeman, L.R.J. Hoy, J.B. Rose, and P.A. Staniland. Synthesis and properties of polyaryletherketones. Polymer, 22 (8):1096 - 1103, 1981. 
[5] Rong-Ming Ho, Stephen Z D Cheng, Benjamin S Hsiao, and Kenncorwin H Gardner. Crystal morphology and phase identifications in poly(aryl etherketone)s and their copolymers. 1. polymorphism in pekk. Macromolecules, 27:2136-2140, 1994.

[6] Woo Il Lee and George S. Springer. A Model of the Manufacturing Process of Thermoplastic Matrix Composites. Journal of Composite Materials, 21 (11):1017-1055, 1987.

[7] Ali Yousefpour, Mehdi Hojjati, and Jean-Pierre Immarigeon. Fusion Bonding/Welding of Thermoplastic Composites. Journal of Thermoplastic Composite Materials, 17(4):303-341, 2004.

[8] Arthur Levy, Steven Le Corre, and Irene Fernandez Villegas. Modeling of the heating phenomena in ultrasonic welding of thermoplastic composites with flat energy directors. Journal of Materials Processing Technology, 214 (7):1361-1371, 2014.

[9] C A Butler, R L Mccullough, R Pitchumani, and J W Gillespie Jr. An analysis of mechanisms governing fusion bonding of thermoplastic composites. Journal of Thermoplastic Composite Materials, 11(4):338, 1998.

[10] R. P. Wool and K. M. O'Connor. A theory of crack healing in polymers. Journal of Applied Physics, 52(10):5953-5963, 1981.

[11] Young Hwa Kim and Richard P Wool. A Theory of Healing at a PolymerPolymer Interface Young. Macromolecules, 16:1115-1120, 1983.

[12] R P Wool, B.-L. Yuan, and O J McGarel. Welding of Polymer Interfaces. Polymer Engineering and Science, 29(19):1340-1367, 1989.

[13] L. J. Bastien and J. W. Gillespie. A non-isothermal healing model for strength and toughness of fusion bonded joints of amorphous thermoplastics. Polymer Engineering \& Science, 31(24):1720-1730, 1991. 
[14] Rachid Smail, Christian Carrot, and Jacques Guillet. Physically meaningful discrete relaxation spectrum: Rheological behaviour of monodisperse polymer melts. Macromolecular Theory and Simulations, 5:645-661, 1996.

[15] John D Ferry. Viscolelastic Properties of Polymers. John Wiley and Sons, 1980.

[16] Gilles Regnier and Steven Le Corre. Modeling of Thermoplastic Welding. In Nicolas Boyard, editor, Heat Transfer in Polymer Composite Materials: Forming Processes, chapter 8, pages 235-263. John Wiley \& Sons, Hoboken NJ, 2016.

[17] Jean-Florent Lamethe, Pierre Beauchene, and Liliane Leger. Polymer dynamics applied to PEEK matrix composite welding. Aerospace Science and Technology, 9(3):233-240, 2005.

[18] Julien Avenet, Arthur Levy, Jean Luc Bailleul, Steven Le Corre, and Jerôme Delmas. Adhesion of high performance thermoplastic composites: Development of a bench and procedure for kinetics identification. Composites Part A: Applied Science and Manufacturing, 138(July), 2020.

[19] F. Yang and R. Pitchumani. Healing of thermoplastic polymers at an interface under nonisothermal conditions. Macromolecules, 35(8):3213-3224, 2002.

[20] F Yang and R Pitchumani. Nonisothermal Healing and Interlaminar Bond Strength Evolution During Thermoplastic Matrix Composites Processing. Polymer Composites, 24(2):263-278, 2003.

[21] T. Choupin, B. Fayolle, G. Régnier, C. Paris, J. Cinquin, and B. Brulé. Macromolecular modifications of poly(etherketoneketone) (pekk) copolymer at the melting state. Polymer Degradation and Stability, 155:103 $110,2018$. 
[22] Tangauy Choupin. Mechanical performances of PEKK thermoplastic composites linked to their processing parameters. PhD thesis, l'École Nationale Supérieure d'Arts et Métiers, 2017.

[23] Emilie Courvoisier, Yoann Bicaba, and Xavier Colin. Multi-scale and multitechnique analysis of the thermal degradation of poly (ether ether ketone). Polymer Degradation and Stability, 151:65-79, 2018.

[24] Francisco Sacchetti, Wouter J.B. Grouve, Laurent L Warnet, and Irene Fernandez Villegas. Effect of resin-rich bond line thickness and fibre migration on the toughness of unidirectional Carbon/PEEK joints. Compos. Part A Appl. Sci. Manuf., 109:197-206, 2018. 